\title{
INTELLIGENT GRID DATA MANAGEMENT IN CLOUD COMPUTING ENVIRONMENT
}

\author{
B. Mallikarjuna ${ }^{1}$, A. Nagaraju ${ }^{2}$ \\ ${ }^{1}$ Assistant Professor in Dept of CSE in GITAM University, Telangana, India \\ ${ }^{2}$ Assistant Professor in NBKR Institute of Science and Technology, Vidyanagar, Nellore, Andhra Pradesh, India
}

\begin{abstract}
An Intelligent grid data management is one of the upcoming future of the integrated and optimization of power and data management, it is having two-way approach of optimization power and data. The balancing factors of power, and optimization of data is useful to create an automatic to the intelligent grid data management. In this paper we discussed in cloud computing environment how integrated architecture of intelligent grid data management works and utilize power and data, our aim is to obtain the optimized intelligent grid data management and balancing factors of power grid and data management in this mechanism we named as Integrated architecture of an intelligent grid data management in Cloud computing. The intelligent grid mechanism to manage the balancing of power usage cost and optimization of data in grid utilization. The simulation result shows this mechanism is reduce the power consumption and efficient data utilization mechanism in intelligent grid management compared to non intelligent grid management and effective result then intelligent grid data management in cloud computing environment.
\end{abstract}

Keywords: Intelligent grid data management, Power consumption, balancing factors of power, optimization of data, power grid, grid utilization, cloud computing.

\section{INTRODUCTION}

An intelligent grid is upcoming features of the electrical grid in information and communication technology to obtain the information and act on information about the behaviors suppliers and consumers have effective utilization of power and data to improve the efficiency, reliability, availability, cost. To manage the balancing factors of efficiency, reliability, availability and cost to control production and distribution of electricity and data are important aspects of the intelligent grid, optimization of functionality of their power and data generation, transmission, distribution, demand in intelligent grid. In power grid the important element are digital processing and communication, present an intelligent grid data flow and information management central to the intelligent grid. Integrated technology of intelligent grid are making the key issues of various capabilities. In power grid the important element are digital processing and communication, present an intelligent grid data flow and information management central to the intelligent grid. Integrated technology of intelligent grid are making the key issues of various capabilities.

Balancing parameters of Intelligent Grid Data Management there are the integrated fashion through various factors are reliability, efficiency, availability, cost, flexibility in network topology, efficiency, load adjustment or load balancing, peak curtailment or Leaving and time of using pricing, sustainability, market and enabling, demand and response support, plat form advanced services and many other parameters, here some of the primary fallowing parameters are discussed in this paper.
Optimization methodology in intelligent grid data management of making optimize such as power and data generation, transmission and distribution to design such as fully minimum functionality. Power and data generation constraints to maintain adequate online reserves, minimization of the size of data and unit power of uploading data item in cloud. Transmission line congestion limits to prevent overloads, optimization of the data manipulations and intermediate transmission between one cloud to another cloud. Incremental heat rate characteristics for each generation unit. Detailed nonlinear cost function modeling in demand optimization to optimize the data redundancy, task execution, data splitting, data exclusive, data upload and inter and intra cloud data transfer constraints.

This paper is organized as follows. Section 2 discuss about the related architecture of intelligent grid data management in cloud computing. Section 3 describes the integrated architecture of intelligent grid data management Section 4 presents possibilities of optimization techniques on the intelligent grid data management. Section 5 deals with the simulation results and experimental analysis and finally, conclusions are drawn in Section 6.

\section{THE ARCHITECTURE OF INTELLIGENT} GRID DATA MANAGEMENT IN CLOUD COMPUTING

In this section we are going to discuss the how cloud computing is interacted with the intelligent grid. There are various cloud providers such as Amazon, Microsoft and 
Google having the huge and large amount of datacenters[2]. Amazon having the biggest datacenter in United Sates[3]. Every datacenter consist of the servers, cooling machines, power management, power transformers[1,2,3,4]. Microsoft datacenter in quinsy, Washington having 43,602 square meters of space and uses $4.9 \mathrm{KM}$ of chillier pipeline, 966 $\mathrm{KM}$ of power wire 92,900 squares meters of drywall and 1.5 metric tons backup batteries[1,2,3,4]. The fallowing figure 1 virtual diagram shows the how the datacenters are interacted with the cloud computing is communicated with in intelligent Grid

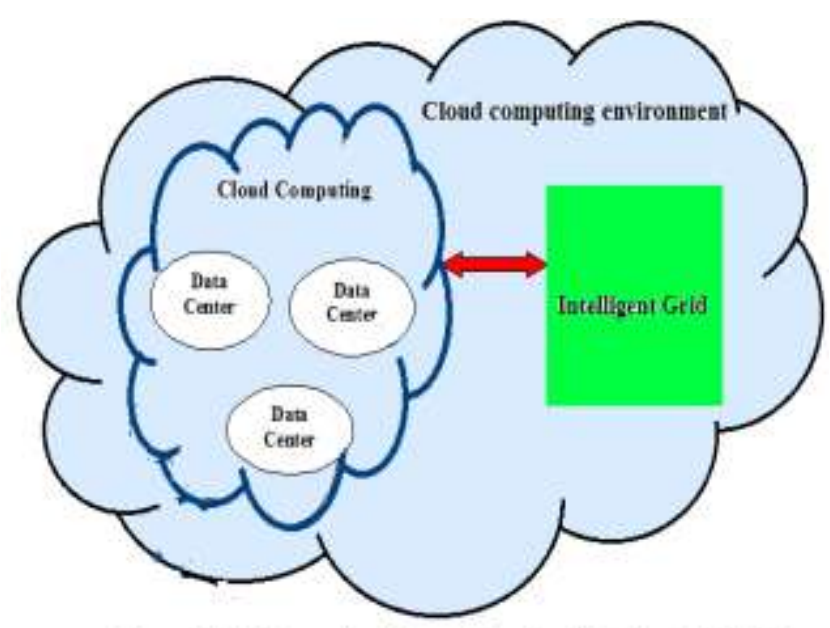

Figure 1: The interaction between Cloud and Intelligent Grid [4 ].

The interaction between clod and intelligent grid is done through the various massively huge number of datacenters as shown in figure 1 . The datacenters in cloud computing plays a major role on the primary balancing factors of power grid such as efficiency, reliability, availability and cost. Datacenters are the heart of the cloud computing and requires the huge amount of the electricity if datacenters are increasing electricity and cost increasing.

Power Grid: The power grid was designed and implemented 120 years ago, the power grid is integrated on intelligent grid, electricity power is produced at the power generator from power plant it consist of the transformers. This power generation plants are very large in infrastructure and are located far away from huge populated areas [12]. The power generator connects to the generator setup transformer it transform the higher voltage electricity power through the transmission lines for long distance to reach the substation set up transformer. Then after reaching the substation setup transformer the higher voltage electricity power distributed voltage and transform to the substation, primary and secondary customers where it will be located as shown in figure 2.

In cloud Computing how to interact the data management on intelligent grid. Data Management system having the storage, integration, validation analysis and increase the performance of different data in intelligent grid [4]. The cloud provider provides the service provider and end user, the service provider is nothing but intelligent grid data management as shown in Figure 2. The intelligent grid data management under service provider consist of the four sub domains such as power and data generations, transmission, distribution, customers. Under end user consist of the market and services and operations. The service provider act as intelligent grid, it will provide all services and fulfill the end user. The end user asks for solutions to the cloud provider cloud provider provides the service as shown in figure 2 .

Intelligent Grid Data Management: An Intelligent grid is the integrated architecture of the power grid in cloud environment it divided into three modules such as cloud provider, service provider and end user. The service provider it act like heart of intelligent grid it contains power and data generation, transmission, distribution, customers, market, service and operation. The power grid is integrated through the intelligent grid, the balancing factors of efficiency, reliability, availability and cost and optimizing the generation, distribution, transmission and demand. The integrated architecture of intelligent grid data management as shown in figure 2 .

Cloud Provider: It will provide the environment where services are performed their execution. All user are connected through the cloud provider. The user submitted applications or developed the applications submitted to the cloud provider, cloud provider allocate the resource to different requests and different requirements.

Service Provider: It provides organizations with consulting, legal, real estate, education, communications, storage, processing, and many other services. Such a process of allocation and reallocation of resources is the key accommodating unpredictable demands and improving the return on investment from the infrastructure supporting to the cloud.

End User: In intelligent grid the end user is the individual who uses the service by the demand for the cloud computing.

Power and Data Generation: In intelligent grid power and data generation is a concept of matching electricity and data production with demand using multiple identical generators which can start, stop and operate efficiently at chosen load, independently of the others, the load of the generation it may vary or it may stable make them suitable for base load and peaking power and data generation. Matching power and data supply and demand, called load balancing, is essential for a stable and reliable supply of power and data. The load balancing depends up on various factors short-term deviation in the balance lead to variations of frequency and a prolonged mismatch result in blackouts, transformers to store the huge amount of power for the future use.

Transmission: The broadcasting of electric power and data transmission, electricity and data distribution from one location to another, as from a transmitter to a receiver. The intelligent grid is a centralized unidirectional system. 
Distribution: The process of making electricity or data and service available for use or consumption by a consumer.

Customers Demand: An individual who uses of electricity or data, may store the power or data and manage the electricity power or data, the customer who pays for supply and creates demand.

Market: In intelligent grid environment market can be called the manage electricity or data on available or current existing electricity power that of all existing and supplying needs.
Services: An intelligent grid environment it use of robust two-way communications, advanced sensors, and distributed computing technology will improve the efficiency, reliability and safety of power delivery and use of data. It also opens up the potential for entirely new services or improvements on existing ones, such as fire monitoring and alarms that can shut off power, make phone calls to emergency services, etc.

Operations: End user required all above operations with various deployment to new ways to manage supply and demand of integrated communications.

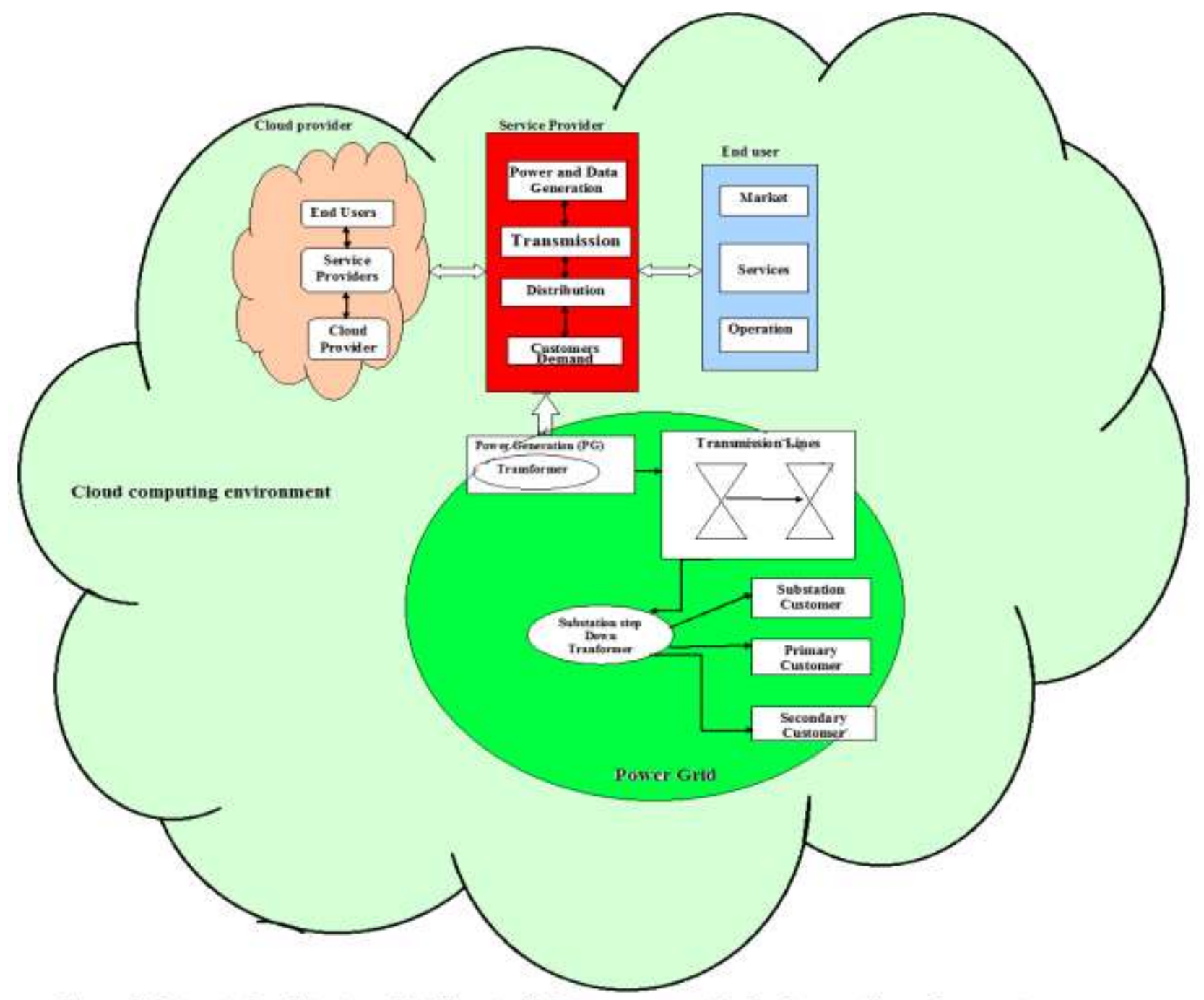

Figure 2: Integrated architecture of intelligent grid data management in cloud computing environment

The cloud provider demands the service to the service provider, service provider consist of large amounts of data storage and provide service for appropriate request for cloud provider. The services are many types if service are exist it may be new service as for parent child process. The resource provisioning concept, allocate resources among the best process is also challenging task. This really advances the use of resources and services for different participants in the intelligent grid environment management.

\section{OPTIMIZATION TECHNIQUES ON INTELLIGENT GRID DATA MANAGEMENT}

An integrated architecture of intelligent grid consist of the service provider and power grid, the total load connected to the intelligent grid may vary significantly over time. Even though the total load is the sum of many individual choices of the clients, the overall load is may be a stable, may be varying. Suppose for example increment of the load if a popular television program starts and millions of televisions 
will draw current at once, usually to respond a rapid increase in power consumption, faster than the start-up time of a large generator, some spare generators are put on a dissipative standby mode. It requires the balancing factors of efficiency, reliability, availability and cost of intelligent grid to warn all individual television sets, or another larger customer, to reduce load temporarily or continuously. .

\section{Balancing Factors of the Power Grid}

Algorithm 1: Balancing factors in intelligent grid data management

Begin

Input: Efficiency, Reliability, Availability, Cost output: balancing Efficiency, Reliability, Availability and Cost

Step 1: After initialize power grid PG.

Step 2: Calculate the distribution of power $P D$ approaching cloud services initializing the cost function for billing the power balancing factor from step 3 .

Step 3: Efficiency output power delivered to the load of grid and $P_{\text {in }}$ is total input power

Step 4: Availability $\frac{1}{A-25}$ where MTBF is Mean Time Between Failure and MTTR is Mean Time Rate.

Step 5: Reliability $\boldsymbol{R}^{-x} \boldsymbol{R}_{(t)}$ is reliability, e exponential, $\lambda$ failure rate, $\mathrm{m}$ is mean time between failure and $\mathrm{t}$ is time $m=\frac{1}{\lambda}$ i.e

$$
m=\frac{t}{\log _{(n)}\left(\frac{1}{R(t)}\right)}
$$

Step 6: The cost of the Power Grid at various levels $\mathbb{R}_{i} \bar{i}=12 \pi$ minimize the cost of the power grid $P D$ is power distribution of the power grid to balancing factor of the power The unit production cost function is approximately quadratic equation where $a_{i}, b_{i}, c_{i}$ are constants

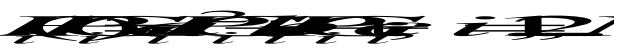

Step 7: Assign cost function to the cloud service providers

Step 8: Repeat Step 3-7 to analyze the details of cost function data by the service providers to estimate the balancing factor $\phi$
Step 9: Compute the power utilization and data of the grid $\boldsymbol{P}_{\boldsymbol{C} \text { on }}=\boldsymbol{\phi}_{\boldsymbol{u}_{d}}$ where $\boldsymbol{\varphi}_{\text {is }}$ the balancing factor, $u_{d}$ is the utilization of the devise and $c_{d}$ is the capacity of the device for each device consumption in grid do occurrences power utilization and data take back power utilization and data amass power utilization and data

End

Step 10: repeat all above steps until the details of power consumption to the intelligent grid service providers End

\section{Optimization Technique on Intelligent Grid}

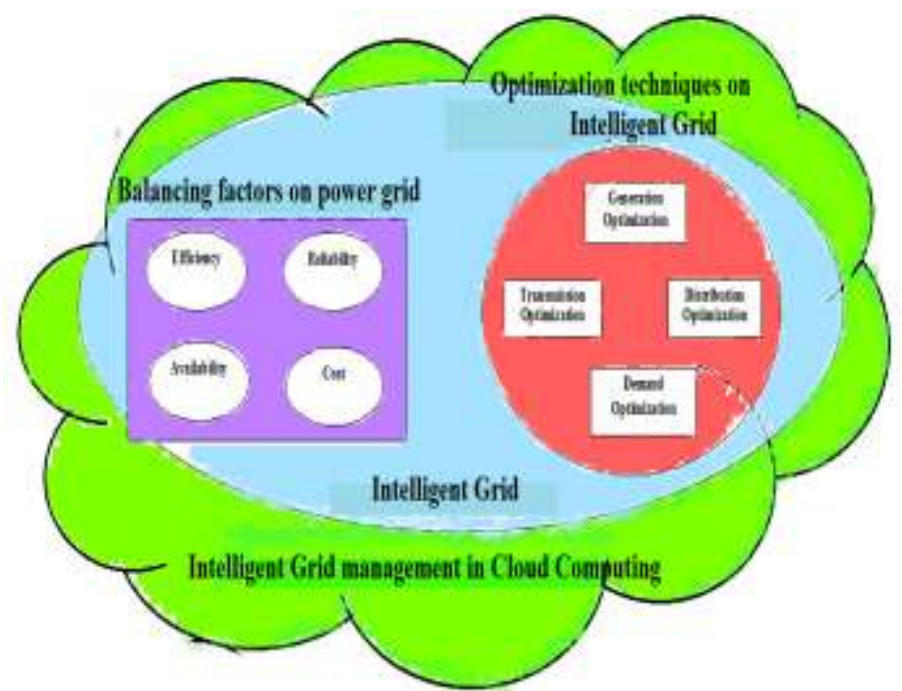

Figure 3: The primary internal factors of an intelligeat grid data management in chud computing

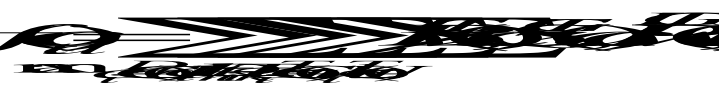

In generation optimization there are number of clouds in cloud environment set of clouds there are number of datacenters required the set of data items Che the set of to calculated the summations are $a_{1}, a_{2}, a_{3}$. Unit size of data items storage at datacenter in cloud $S_{Z}{ }^{D}(d, c)$ and unit storage power required at datacenter in particular cloud $\mathrm{c}$ is $P \boldsymbol{w}^{s t}(c)$, power of uploading data item at datacenter in cloud is $\boldsymbol{P W}^{\boldsymbol{N}}(\boldsymbol{d}, \boldsymbol{c})$, power of downloading data item at datacenter in cloud $P w^{D}(d, c)$.

The generation optimization to calculate the overall cost of

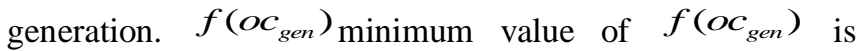

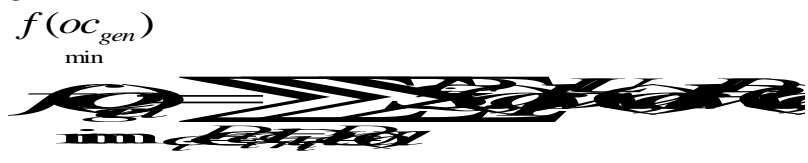

(1) 
In transmission and distribution optimization the particular task $t$ the set of tasks $T_{k}$ is of cost binary variable for uploading the data at datacenter in cloud $B \boldsymbol{v}^{U \mathbb{L}}(\boldsymbol{d}, \boldsymbol{c})$ with the unit storage power required at datacenter in cloud $P w^{S t}(c)$, binary variable task $t$ to cloud $c$ is $B r^{7 k}(t, c)$, the computation cost charged by task $t$ to cloud $c$ is $C p(t, c)$, The cost of is the Binary variable integrated transmitting tasks $t_{1}, t_{2}$ cloud $c_{1}, c_{2}$ is

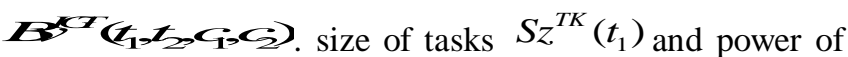

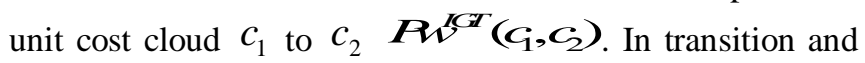
distribution optimization to calculate the overall cost of transition and distribution, the overall cost $f\left(o c_{t d}\right)$ maximum value of $f\left(o c_{t d}\right)$ is $f\left(o c_{t d}\right)$

In transition and distribution optimization, the objective function of each and every task is executed in the cloud.

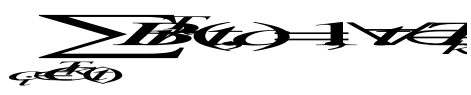

In demand optimization domain most utilities rely on manual processes spread sheets and independent software applications to decide if, when, and how many resources are needed to support forecasted demand. the system's demand dispatch support tool recommendations to the use for optimal results. storage splitting ratio at datacenter of data item $\vartheta(d)$, data redundancy ratio at datacenter $\mu(d)$ as inequality constraint size of data item is less then ratio of the data, the objective functions are defined as fallows, the demand optimization is minimum

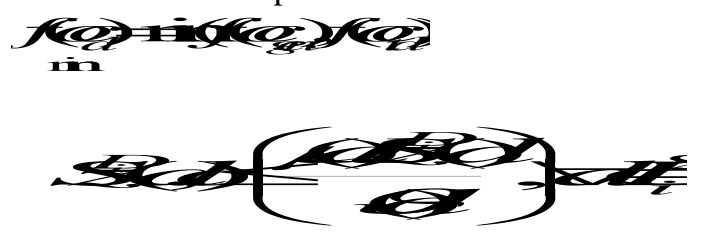

size of data item in cloud the ratio storage data redundancy with in $\{0,1\}$

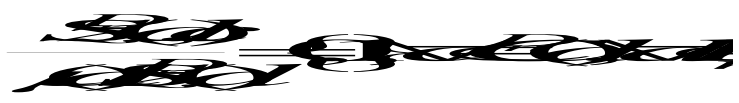

eq.(13) is data exclusive constraint then

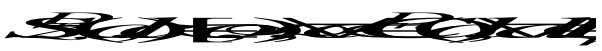

In demand data binary variable data upload to cloud constraint

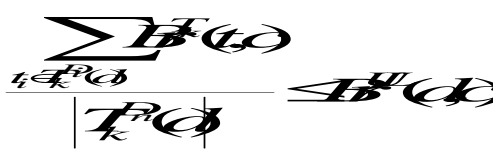

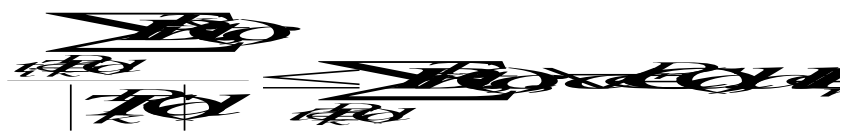

(8)

Binary variable the task performing at inter cloud

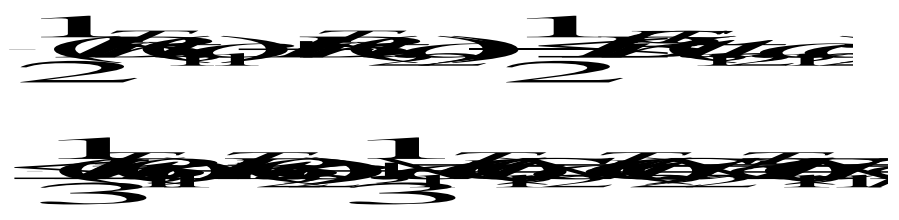

\section{RESULTS AND DISCUSSION}

The results of an Intelligent Grid Data Management which operates for number of hours in a day at load balancing transmission. we brought the data from the reliability of test system through the website [2]. we shown the simulation result lot of betterment then the intelligent grid management and non intelligent grid management as shown in figure 4(a)(b).

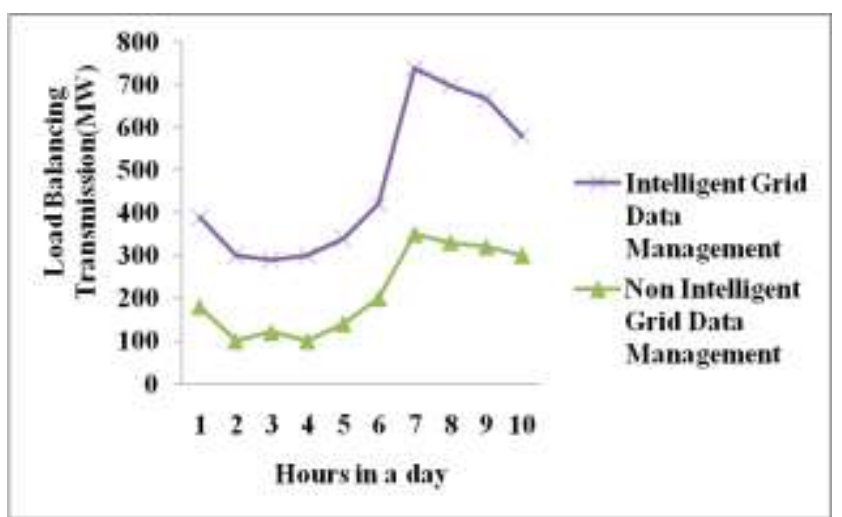

Fig 4: a) Hours in a day observation load balancing transmission of intelligent grid

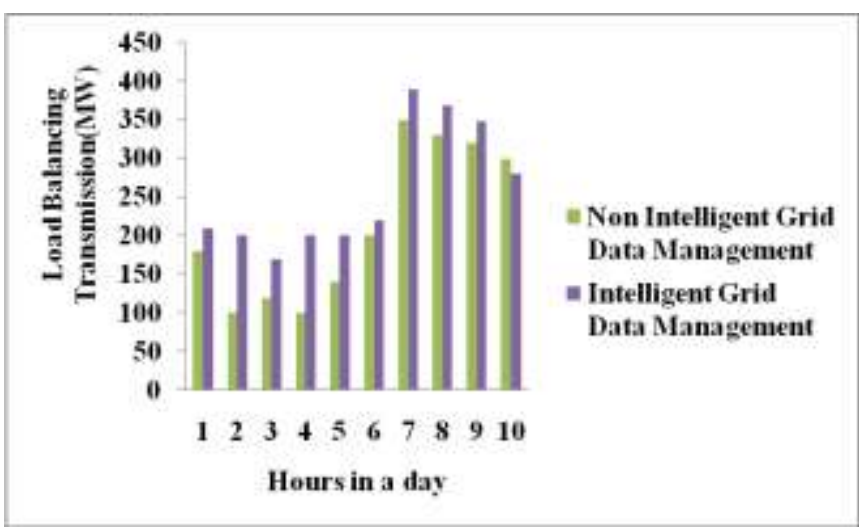

Fig 4b) load balancing transmission increasing the peak hours.

An intelligent grid data management is tested with the MATLAB,R2011b version. The mathematical model of optimization power and data management as shown figure 5(a) (b). 


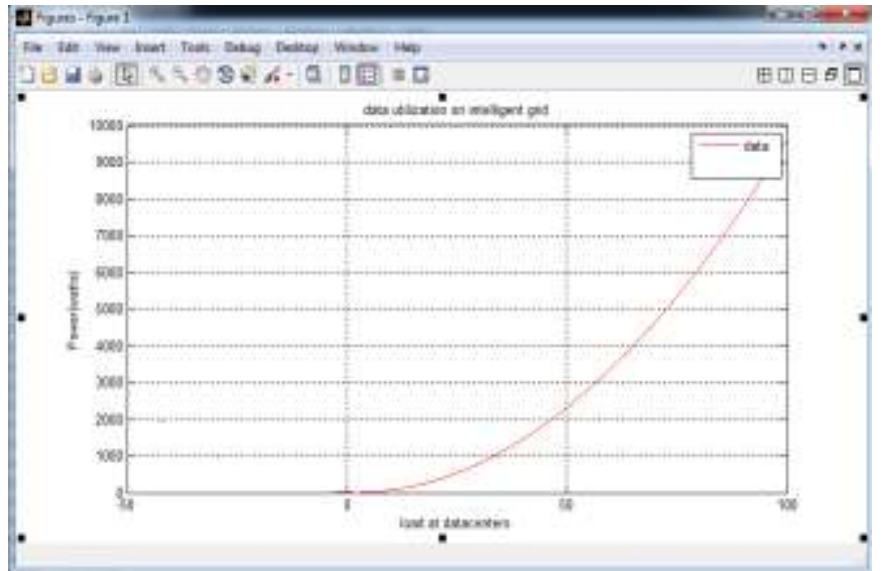

Fig 5a) data utilization on intelligent grid

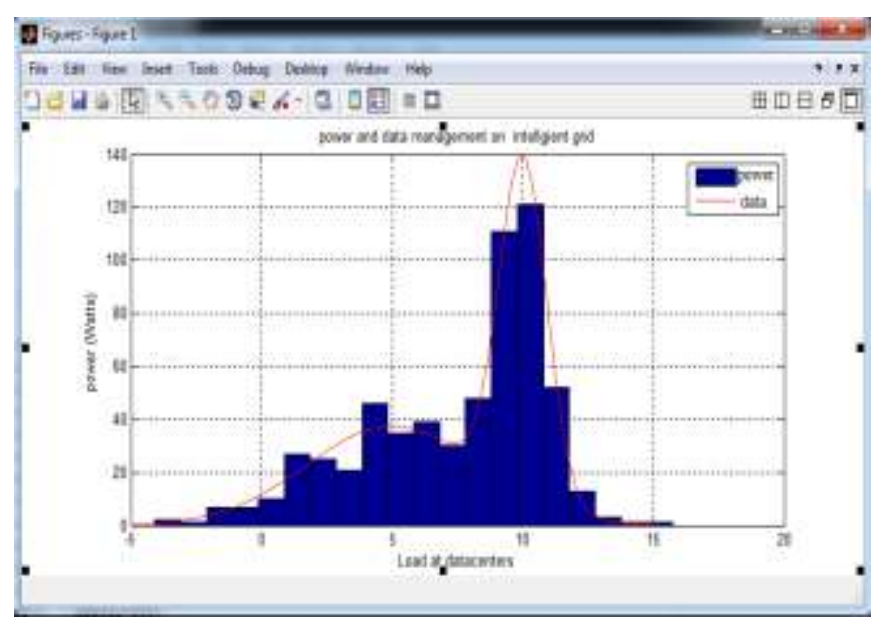

Fig 5b) power and data utilization on intelligent grid

\section{CONCLUSION}

This paper presents the power and data management mechanism for intelligent grid. It interacts with the huge and massive datacenters to provide communication between intelligent grid with integrated architecture in cloud computing environment, we concentrated on design possibility that can balance the power load and data management on the intelligent grid by distributing the service requests among the datacenters. The optimization methods provides analysis for power utilization and effective utilization of data management in cloud computing, still there are many issues to solve. This simulation results provide the effective management of data and power on intelligent grid, we implement optimization techniques on various tool kit to measure the effective results. The future work to implement optimization techniques in intelligent grid and micro grid.

\section{REFERENCES}

[1]. L.D. Dhinesh Babu, P.V Krishna et. " An Analysis of Security Related Issues in Cloud Computing ", CONTEMPORARY COMPUTING, Communicatons in Compter and Information Science, 2011. Part 2, 180-190.

[2]. "Reliability Test System Task Force of the Application of Probability Methods subcommittee- The IEEE Reliability Test System-1996," pp.1010-1020, Aug.1999
[3]. X.Fan, W.D Weber, and L.A. Barroso, "Power provisioning for a warehouse sized computer ", in ACM International Symposium on Computer Architecture, San Diego, CA, Jun. 2007.

[4]. B. Heyes, "Cloud Computing ", Communications of the ACM. vol.51.,no 7,pp. 9-11, Jul.2008.

[5]. R. Buyya et al., " Cloud Computing and emerging it platforms: Vision, Hype, and Reality for Delivering Computing as the $5^{\text {th }}$ Utility, Future Generation Comp. Sys., 2009, pp. 599-616.

[6]. B. Mallikarjuna, P.V Krishna " Adversary Search technique on critical workflow tasks in Load balancing of tasks in Cloud computing" National Conference RAECE IIT Roorkee, Feb 13-15, 2015.

[7]. Gon, calves, G.E et al., "Resource Allocation in Clouds: Concepts, Tools and Research Challenges", XXIX Brazilian Symposium on Computer Networks and Distributed Systems, 2011.

[8]. S. Misra, P.V Krishna, K. Kalaiselvan, V. Saritha and M.S. Obaidat, " Learning Automata-Based QoS Framework for Cloud IaaS", IEEE Transactions on Network and Service Management, Vol. 11, Issue. 1, pp. 15-24,2014.

[9]. L.D.D Babu, P. Venkata Krishna, "versatile time-cost algorithm (VTCA) for scheduling non- preemptive tasks of time critical workflows in cloud computing system", Int. J. Communication networks and Distributed Systems, Vol. 11, No. 4, 2013.

[10]. L.D.Dhinesh Babu and P Venkata Krishna, "Honey Bee Behavior inspired Load Balancing of tasks in Cloud Computing Environments", Applied Soft. Computing, Volume 13 Issue 5, Pages 2292-2303 Elsevier, May, 2013

[11]. Buyya, R.; Ranjan, R.; Calheiros, R.N.; Modeling and simulation of scalable Cloud computing environments and the CloudSim toolkit: Challenges and opportunities, High Performance Computing \& Simulation, 2009. HPCS '09. International Conference on, pp.1-11, 2009.

[12]. D Karaboga " An Idea Based on Honey Swarm for Numerical Optimization" Technical Report- TR06, Oct, 2015.

[13]. B.Mallikarjuna, P. V Krishna. "A Nature Inspired Approach for Load Balancing of Tasks in Cloud Computing using Equal Time Allocation" Communicated International Arab Jounal of Information Technology (IAJIT) Dec 2014.

[14]. B.Mallikarjuna, P.V Krishna. "OLB: Nature Inspired Approach for Load balancing of tasks" commmunicated Degruyter Journal Feb 2015.

[15]. G,Bruce Berriman,Ewa Deelman,Paul Groth and Gideon Juve " The Applications of Cloud Computing to the Creation of Image Mosaics and Management of their PRovenance" astocompute.wordpress.com.

[16]. Gideon Juve, Ewa Deelman etc.."Scientific Wokflow Applications on Amazon EC2"Cornell University Library http://arxiv.org/pdf/1010.4822.pdf.

[17]. EXELIS visual information solutions http://www.exelisvis.com/docs/MosaicImages.html.

[18]. Amazon.com, "Elastic Block Store (EBS)"; http://aws.amazon.com/ebs.

[19]. Amazon.com. "Simple Storage Service(S3)"; http://aws.amazon.com/s3. 
[20]. Krishna, P.V.; Misra, S.; Joshi. D.; Obaidat, M.S.; Learning Automata Based Sentiment Analysis for Recommender System on Cloud, 2013 IEEE International Conference on Computer, Information, and Telecommunication Systems, CITS 2013, May 7-8, 2013.

[21]. Priya Pandey, P Venkata Krishna, B Sarojini, "QoS Aware Healthcare System on Mobile Clouds", World Congress on Computing and Communication Technologies (WCCCT), 154-157, IEEE, 2014.

[22]. P.M.Devie, S.Kalyani "An Optimization Framework for Cloud-Based Data Management Moel in Smart Grid" International Journal of Research in Engineering and Technology (IJRET) Vol 04 Issue 02 Feb 2015.

[23]. Chenhong Zhao, Jain Xie "Independent Tasks Scheduling Based on Genetic Algorithm in Cloud Computing" Sponsored by National Innovative project 9781-4244-3693-4/09 IEEE 2009.

[24]. GAN Guo, HUANG Ting-lei, GAO Shuai "Simulated Annealing Algorithm for Task Scheduling based on Cloud Computing Environment" 978-1-4244-6837-9 IEEE 2010.

[25]. Mahdi Mehrshad, et.. at "Demand Side Management in Smart Grid Data Based on Energy Consumption Scheduling by NCGA-II " International Journal of Engineering and Practical Research (IJEPR), Vol 2 Issu4, Nov 2013.

[26]. Kennedy J, Eberhart R, et al. "Particle swarm optimization" Proceedings of IEEE international conference on neural networks, vol. 4. Piscataway, NJ: IEEE; pp. 19421948, 1995.

[27]. K. Deb, Multi objective Optimization using Evolutio nary Algorithms, John Wiley \& Sons, UK, 2001.

[28].http://www.smartgridnews.com/artman/publish/Technol ogies_Demand_Response/

[29]. "Reliability consideration from the integration of smart grid" North American Electric Reliability Corporation (NERC) Dec 2010. www.nerc.com

[30]. Amit Konar " Artificial Intelligence and Soft Computing Behavioural Cognitive Modelling of the Human Brain" pp. 449-473.

\section{BIOGRAPHIES}

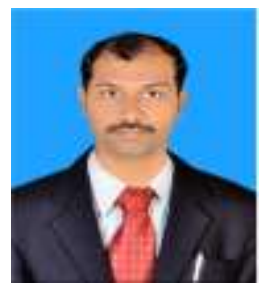

B.Mallikarjuna, Is Assistant Professor in GITAM Institute of Technology, GITAM University Hyderabad, India. $\mathrm{He}$ received his B.Tech Degree in Computer Science and Engineering from JNTU Hyderabad India. M.Tech Computer Science and Engineering from VIT University, Vellore Tamilnadu, India. His research Interests Mobile Computing, Soft Computing, Ad hoc Networks and Artificial Intelligence. He is the member of Institute of Engineers India and IEEE

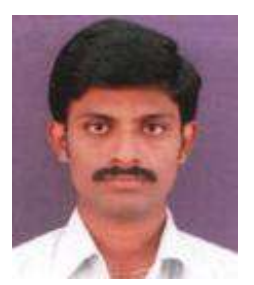

A. Nagaraju, Is currently working as Assistant Professor of Computer Science and Engineering in NBKR Institute of Science and Technology. He received his B.Tech Degree in Computer Science and Engineering from J.N.T.U University Hyderabad Andhra Pradesh India. M.Tech
Degree in Computer Science and Engineering from Bharath Institute of Science and Technology Tamilnadu, India. 